\title{
69. On the Solvability of Goursat Problems and a Function of Number Theory
}

\author{
By Masafumi Yoshino \\ Department of Mathematics, Tokyo Metropolitan University \\ (Communicated by Kôsaku Yosida, M. J. A., June 11, 1981)
}

1. Introduction. In this paper we shall study the reduced Goursat problem with constant coefficients :

$$
L u=\left(a \partial_{1}^{-1} \partial_{2}+\varepsilon+b \partial_{1} \partial_{2}^{-1}+c \partial_{1}^{2} \partial_{2}^{-2}\right) u=h(x)
$$

where $x=\left(x_{1}, x_{2}\right) \in C^{2}, \partial_{i}=\partial / \partial x_{i}(i=1,2)$ and $\partial_{i}^{-1}$ is the integration with respect to the variable $x_{i}$ from the origin to $x_{i}$.

If the roots $\lambda_{1}, \lambda_{2}, \lambda_{3}$ of the characteristic equation of $L$;

$$
a \lambda^{3}+\varepsilon \lambda^{2}+b \lambda+c=0
$$

satisfy the "Alinhac-Leray condition" $\left|\lambda_{1}\right| \leqq\left|\lambda_{2}\right|<\left|\lambda_{3}\right|$ the solvability and the uniqueness of (1.1) are proved by $\mathrm{S}$. Alinhac in [1] under some additional conditions. Whereas, if the condition is not satisfied few results are known. The best work known is that of Leray's for (1.1) with $c=0$ in [2]. He introduced the number-theoretical function $\rho(\theta)$ (cf. [2]) and expressed a sufficient condition for the solvability and uniqueness of (1.1) for $c=0$ in terms of $\rho(\theta)$.

The purpose of this paper is to study the case $c \neq 0$ without assuming the Alinhac-Leray condition. We introduce a function $\rho\left(\theta_{1}, \theta_{2}\right)$ as a natural extension of the Leray's auxiliary function $\rho(\theta)$ which describes the transcendency of $\theta_{1}$ and $\theta_{2}$. In terms of this function we shall characterize the range of the operator $L$. As a result we reveal a close connection between the algebraic-transcendental properties of the characteristic roots and the solvability and uniqueness. We remark that the results here can be extended to a wider class of equations with multiple characteristic roots.

2. Statement of theorems. Without loss of generality we may assume that $a c \neq 0$. Moreover, by the linear change of variables such as $r x_{1}=z_{1}, x_{2}=z_{2}(r \neq 0)$ we may assume that eq. (1.2) has the root 1 and that the absolute values of other roots do not exceed 1 . Since we are interested in the case where the Alinhac-Leray condition is not satisfied we assume $0<\left|\lambda_{1}\right| \leqq\left|\lambda_{2}\right|=1$. Let $H_{0}$ be the set of functions analytic at the origin. Then

Theorem 2.1. If the roots $\lambda_{1}, \lambda_{2}, 1$ of eq. (1.2) are not distinct the map $L: H_{0} \rightarrow H_{0}$ is bijective.

In view of this theorem we shall consider the case where the roots $\lambda_{1}, \lambda_{2}, 1$ are distinct. Let $I_{k}$ be defined by 
Then we have

$$
I_{k}=\lambda_{1} \lambda_{2}\left\{\lambda_{1}^{k+2}\left(1-\lambda_{2}\right)+\lambda_{2}^{k+2}\left(\lambda_{1}-1\right)+\lambda_{2}-\lambda_{1}\right\}
$$

Proposition 2.1. The map $L: H_{0} \rightarrow H_{0}$ is injective iff $I_{k}$ does not vanish for $k=1,2, \cdots$.

To study the range of $L$ we consider the following three cases; A) $\left|\lambda_{1}\right|=\left|\lambda_{2}\right|=1$, B) $\left|\lambda_{1}-1\right|=\left|\lambda_{1}-\lambda_{2}\right|$ and $\left|\lambda_{1}\right|<1$, C) otherwise.

Case A) Write $\lambda_{j}=\exp \left(2 \pi i \theta_{j}\right), 0 \leqq \theta_{j}<1(j=1,2)$ and define the function $\rho\left(\theta_{1}, \theta_{2}\right)$ by

$$
\rho\left(\theta_{1}, \theta_{2}\right)=\liminf \inf _{k \rightarrow \infty}\left(\left|k \theta_{1}-p\right|^{1 / k}+\left|k \theta_{2}-q\right|^{1 / k}\right) .
$$

Note that the function $\rho(\theta, 0)$ is the one introduced by J. Leray in [2]. Then we have

Theorem 2.2. Let $\left(\lambda_{1}, \lambda_{2}\right)$ be in Case A). Then $L H_{0}=H_{0}$ if and only if $\rho\left(\theta_{1}, \theta_{2}\right)>0$.

Remarks. a) It follows from the definition of $I_{k}$ that $I_{k}$ vanishes iff both $\theta_{1}$ and $\theta_{2}$ are rational. Hence, by Theorem $2.1 L$ is bijective iff $\rho\left(\theta_{1}, \theta_{2}\right)>0$.

b) If we define $\Delta$ as the set of all real $\theta$ satisfying $\rho(\theta, 0)=0$ we can see that $m_{1}(\Delta)=0$ and that the set $\Delta$ has the density of continuum (cf. [3]). Then the set of all $\left(\theta_{1}, \theta_{2}\right)$ such that $\rho\left(\theta_{1}, \theta_{2}\right)=0$ are contained in $\Delta \times \Delta$ and contains all the points $(l \theta, m \theta)$ where $\theta \in \Delta$ and $l$ and $m$ are integers.

Next we shall study the case $\rho\left(\theta_{1}, \theta_{2}\right)=0$. First we consider the case where both $\theta_{1}$ and $\theta_{2}$ are rational. We determine the integers $s_{1}$ and $s_{2}$ by $\theta_{1}=r_{1} / s_{1}, \theta_{2}=r_{2} / s_{2}$ where $r_{1}, s_{1}$ and $r_{2}, s_{2}$ are relatively prime non-negative integers respectively. We denote the least common multiple of $s_{1}$ and $s_{2}$ by $s_{0}$. Then

Theorem 2.3. A function $h(x) \in H_{0}$ is in the image $L H_{0}$ of $H_{0}$ by $L$ iff $h(x)$ satisfies, for all $k=s_{0} p-1, s_{0} p-2(p=1,2, \cdots)$,

$$
\sum_{j=1}^{k} h_{k-j, j-1} I_{k-j}=0
$$

where $h(x)=\sum h_{p, q} x_{1}^{p} x_{2}^{q} /(p ! q !)$. The kernel of the map $L: H_{0} \rightarrow H_{0}$ is an infinite-dimensional vector space.

To study the case where either $\theta_{1}$ or $\theta_{2}$ is irrational we need some preparations.

For each $\eta \geqq 0$ we define the class of entire functions $B_{\eta}$ by

$$
B_{\eta}=\left\{h \in H_{0} ;\left|h_{\alpha}\right| \leqq M_{0} r_{1}^{|\alpha|}\left(\alpha_{1} ! \alpha_{2} !\right)^{1-\eta} \text { for some } M_{0}, r_{1}>0\right\} \text {. }
$$

Here $h(x)=\sum h_{\alpha} x^{\alpha} / \alpha$ !. Note that $B_{0}=H_{0}$. Let $t=\left[a_{1}, a_{2}, \cdots\right]$ be a continued fraction expansion of irrational number $t(0<t<1)$ with

$$
a_{1}=[1 / t], \quad \alpha_{2}=1 / t-a_{1}, \cdots, a_{n}=\left[1 / \alpha_{n}\right], \quad \alpha_{n+1}=1 / \alpha_{n}-a_{n},
$$

where $[\mu]$ denotes the largest integer $\leqq \mu$. Then we determine the integer $q_{n}(n=1,2, \cdots)$ by the relation $q_{n}=a_{n} q_{n-1}+q_{n-2}, q_{-1}=0, q_{0}=1$ $(n=1,2, \cdots)$ and set, for $\gamma \geqq 0$, 
$J_{\gamma}=\left\{t ; 0<t<1, t\right.$ is irrational and satisfies $\left(a_{n+1}\right)^{1 / q_{n}}=O\left(q_{n}^{\gamma}\right)$ as $\left.n \rightarrow \infty\right\}$. Here if $\gamma=0$ we understand that $O\left(q_{n}^{\gamma}\right)=O(1)$. We easily see that $J_{\gamma^{\prime}}$ $\subseteq J_{\gamma}$ for every $0 \leqq \gamma^{\prime}<\gamma$ and that $J_{\gamma}$ has the density of continuum. Moreover we can prove that $\rho(\theta, 0)=0$ for every $\theta \in J_{\gamma} \backslash J_{0}(\gamma>0)$. Note that $\rho(l \theta, m \theta)=0$ for every $\theta \in J_{\gamma} \backslash J_{0}$ and every integers $l$ and $m$. Then we have

Theorem 2.4. The map $L: H_{0} \rightarrow H_{0}$ is injective and the image $L H_{0}$ has the following properties:

a) Suppose that $\theta_{1}$ or $\theta_{2}$ is in $J_{r}$ for some $\gamma>0$. Then $L H_{0}$ contains $B_{\eta}$ for every $\eta \geqq \gamma$.

b) Let $m_{1}(j=1,2)$ be arbitrary positive integers and let $m_{0}$ $=\min \left(m_{1}, m_{2}\right)$. If $\theta_{j}=m_{j} \theta-\left[m_{j} \theta\right](j=1,2)$ for some $\theta \in J_{\gamma} \backslash J_{\gamma^{\prime}}\left(\gamma^{\prime}<\gamma\right)$ we have

$$
L H_{0} \supseteq B_{\eta} \text { for all } \eta \geqq m_{0} \gamma, \quad L H_{0} \not \supset B_{\eta} \text { for all } 0 \leqq \eta<\gamma^{\prime} .
$$

It follows from b) and the definition of $J_{\gamma}$ that for an arbitrary $\gamma>0$ there exists a set $\Omega_{\gamma} \subset R^{2}$ with the density of continuum such that, for every $\left(\theta_{1}, \theta_{2}\right) \in \Omega_{\gamma}, L H_{0} \supseteq B_{\eta}$ if $\eta \geqq \gamma$ and $L H_{0} \not \supset B_{\eta}$ if $0 \leqq \eta<\gamma$.

Case B) We set $\lambda_{1}=r \exp (\pi i \theta), \lambda_{2}=\exp (2 \pi i \theta)$ where $-1<r<1$. Then

Theorem 2.5. For every $r(-1<r<1)$ there exists a set $F$ of real numbers with $m_{1}(F)=0$ such that if $\theta$ is not in $F$ the map $L: H_{0} \rightarrow H_{0}$ is bijective. Similarly, for every real number $\theta$ there exists a set $\tilde{F}$ $\subset(-1,1)$ with $m_{1}(\tilde{F})=0$ such that if $r$ is not in $\tilde{F}$ the map $L$ is bijective. Here $m_{1}(\cdot)$ denotes the Lebesgue measure in $R^{1}$.

Case C) Let $\left(\lambda_{1}, \lambda_{2}\right)$ be in Case C). Then

Theorem 2.6. Suppose that $I_{k} \neq 0$ for $k=1,2, \ldots$ Then the map $L: H_{0} \rightarrow H_{0}$ is bijective. While if $I_{k}$ vanishes exactly for $k=k_{1}, \cdots, k_{l}$, a function $h \in H_{0}$ is in the image $L H_{0}$ iff $h(x)$ satisfies (2.1) for $k=k_{1}, \cdots, k_{l}$. Furthermore the kernel of $L$ is a finite-dimensional non-trivial vector space.

\section{References}

[1] S. Alinhac: Le problème de Goursat hyperbolique en dimension deux. C. Partial Diff. Eqs., 1(3), 231-282 (1976).

[2] J. Leray: Caractère non Fredholmien du problème de Goursat. J. Math. Pures Appl., 53, 133-136 (1974).

[3] J. Leray et C. Pisot: Une fonction de la théorie des nombres. ibid., 53, 137-145 (1974).

[4] M. Yoshino: On the Solvability of Goursat Problems and a Function of Number Theory (in preparation). 